\title{
Neutrino Physics and Nuclear Astrophysics: the LUNA-MV project at Gran Sasso
}

\author{
Sandra Zavatarelli ${ }^{1}$ \\ I.N.F.N., Sezione di Genova, Italy \\ Via Dodecanneso 33, 16146 Genoa (Italy) \\ E-mail: sandra.zavatarelli@ge. infn.it
}

Solar neutrinos emitted by fusion reactions occurring in the Sun provide a unique and direct way to study the interior of our stars: a precise knowledge of reactions producing neutrinos is mandatory to use these neutrinos as probes. Unfortunately many key reactions are still missing high precision data. The goal of nuclear astrophysics is to provide a deeper knowledge of the nuclear reactions ruling the stellar evolution and the synthesis of the elements of the periodic table. Deep underground in the Gran Sasso laboratory, the cross-sections of the several hydrogen burning reactions have been measured right down to the energies of astrophysical interest by the LUNA (Laboratory for Underground Nuclear Astrophysics) Collaboration. At the end of 2018, a new window to the study of helium and carbon burning will by opened by the installation in Hall B of Gran Sasso of a new 3.5 MV single-ended accelerator (LUNA-MV) able to provide hydrogen, helium and carbon high current beams: this new facility will allow to aggress the study of key reactions shaping the evolution of massive stars towards their final fate. The present contribution is aimed to summarize the main results obtained during the solar phase of LUNA and to highlight their influence on our understanding of the properties of the neutrino and of the Sun. Finally, the future of LUNA during the next decade will be outlined.

EPS-HEP2017, European Physical Society conference on High Energy Physics

5-12 July 2017

Venice, Italy

\footnotetext{
${ }^{1}$ Speaker - on behalf of the LUNA Collaboration

(C) Copyright owned by the author(s) under the terms of the Creative Commons

Attribution-NonCommercial-NoDerivatives 4.0 International License (CC BY-NC-ND 4.0).
} 


\section{LUNA and the solar fusion reactions}

Fusion reaction cross sections shape the element formation in the earliest stages of the Universe (Big Bang nucleosynthesis) and in all the stellar objects formed later on: they control the energy generation and the evolution of stars.

In the Sun, hydrogen is transformed into helium predominantly via the pp cycle, a chain of reactions releasing $26.73 \mathrm{MeV}$, and a small fraction still very uncertain, around $1 \%$, through the $\mathrm{CNO}$ cycle. All these fusion reactions occur in a very well defined energy range, the so-called Gamow peak that in the case of the Sun is typically below $30 \mathrm{keV}$ : as a result, reaction cross sections can be extremely low, down to the femto-barn level, and a direct investigation of thermonuclear reaction rates in a laboratory at the Earth's surface is very difficult because of cosmic background.

The LUNA (Laboratory for Underground Nuclear Astrophysics) collaboration [1] has demonstrated that the low-background environment of an underground laboratory like the Gran Sasso National Laboratory (Italy) is the perfect place to perform direct measurements at the relevant astrophysical energies. LUNA started as a pilot project with a $50 \mathrm{kV}$ accelerator and still remains the only facility in the world running an accelerator deep underground, presently a $400 \mathrm{kV}$ accelerator [2] equipped with hydrogen and helium beams. Common features of the two facilities are the intense beam currents, the long-term stability, and the precise beam energy determination.

The first very relevant LUNA contribution to the neutrino physics was the exclusion of the existence of a possible resonance in the cross section of ${ }^{3} \mathrm{He}\left({ }^{3} \mathrm{He}, 2 \mathrm{p}\right){ }^{4} \mathrm{He}$ at solar energies[3].

As highlighted in Fig. 1a after the $\mathrm{d}(\mathrm{p}, \gamma)^{3} \mathrm{He}$ reaction the pp-chain bifurcates into the pp-I and pp-II/pp-III terminations. The branching between these terminations is constrained to the ratio of the ${ }^{3} \mathrm{He}\left({ }^{3} \mathrm{He}, 2 \mathrm{p}\right){ }^{4} \mathrm{He}$ and ${ }^{3} \mathrm{He}(\alpha, \gamma){ }^{7} \mathrm{Be}$ cross sections: as an increase in the cross section of ${ }^{3} \mathrm{He}\left({ }^{3} \mathrm{He}, 2 \mathrm{p}\right){ }^{4} \mathrm{He}$ would have reduced the branching to the ppII and ppIII the possibility of an undiscovered narrow resonance at energies beyond the reach of early experiments was first raised by Fetisov and Kopysov and Fowler [4] in 1972 as a possible explanation to the solar neutrino deficit. This stimulated the LUNA Collaboration in the 1990's to map the cross-section in the solar Gamow peak with a small home-made $50 \mathrm{kV}$ accelerator. The measurements were extended up to the lower edge of the Gamow peak $(16 \mathrm{keV})$, with an high efficiency detection setup realized with eight Si detectors forming a double squared box: the signature of a fusion event was given by the proton-proton coincidence over a couple of detectors [5]. LUNA put in evidence that the ${ }^{3} \mathrm{He}\left({ }^{3} \mathrm{He}, 2 \mathrm{p}\right){ }^{4} \mathrm{He}$ cross-section is indeed slightly increasing at the thermal energy as expected because of the electron screening effect but there is no evidence at all for any resonance: therefore the possible nuclear physics solution to the solar neutrino problem was ruled out.

The neutrino fluxes from ${ }^{7} \mathrm{Be}$ electron capture reactions and ${ }^{8} \mathrm{~B}$ decay strictly linked the ${ }^{3} \mathrm{He}(\alpha, \gamma)^{7} \mathrm{Be}$ cross section and its depedence on the stellar conditions. This reaction (Q-value = $1.586 \mathrm{MeV})$ is a radiative capture reaction into the ground state or the first excited state $\left(\mathrm{E}_{\mathrm{x}}=\right.$ $429 \mathrm{keV}$ ) of ${ }^{7} \mathrm{Be}$. The ground state of ${ }^{7} \mathrm{Be}$ decays by electron capture into ${ }^{7} \mathrm{Li}$ with a half-life of $53.22 \pm 0.06$, populating in $10 \%$ of decay the first excited state of ${ }^{7} \mathrm{Li}$ at $\mathrm{E}_{\mathrm{x}}=478 \mathrm{keV}$ (deexciting later on with gamma emission to the ground state). The LUNA research activity on 
${ }^{3} \mathrm{He}(\alpha, \gamma){ }^{7} \mathrm{Be}$ was designed in order to exploit two different techniques: in the first approach direct $\alpha$-capture $\gamma$-rays were detected (prompt $\gamma$ method), while, in the second one, the delayed ${ }^{7}$ Be-decay $\gamma$-rays were observed (activation method). Both method gave consistent results as shown in fig. 2 (left) and LUNA was able to achive a high precision result (3\%)[6]. According to [7] the present uncertainty on the ${ }^{7} \mathrm{Be}$ solar neutrino flux is of $7 \%$ being the contribution of the ${ }^{3} \mathrm{He}(\alpha, \gamma)^{7} \mathrm{Be}$ cross section reduced to $4.6 \%$ and of ${ }^{3} \mathrm{He}\left({ }^{3} \mathrm{He}, 2 \mathrm{p}\right)^{4} \mathrm{He}$ to $2.1 \%$. The precision of ${ }^{8} \mathrm{~B}$ neutrino flux is worse, at the level of $14 \%$, being the ${ }^{3} \mathrm{He}(\alpha, \gamma)^{7} \mathrm{Be}$ contribution to the total error budget of only $4.6 \%$. The other relevant contribution to the uncertainty ar in this case the opacity (6.7\%), the diffusion coefficients $(2.8 \%)$ and the ${ }^{7} \mathrm{Be}(\mathrm{p}, \gamma)^{8} \mathrm{~B}$ cross section $(7.7 \%)$.
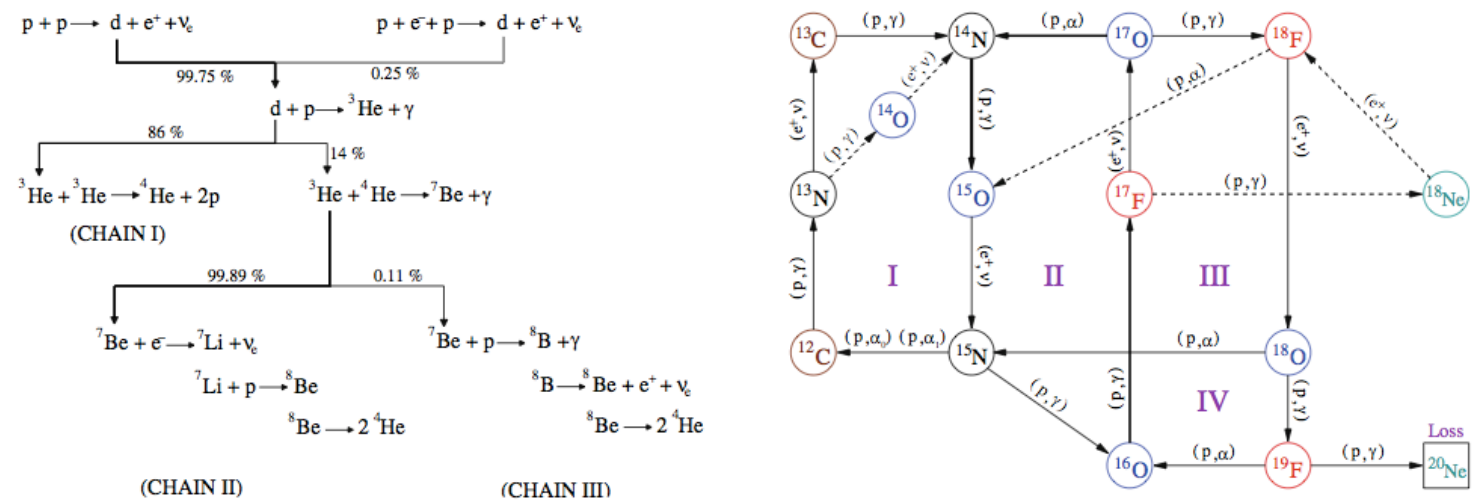

Fig.1 Solar fusion reaction: (left) pp-chain and (right) CNO cycle (for details see [3])

Though the CNO cycle is expected to provide a minor contribution $(\sim 1 \%)$ [7] to the nuclear energy generation in the Sun (with the proton-proton chain being the dominant source), a substantial flux of neutrinos is released by the ${ }^{13} \mathrm{~N}(\beta+v){ }^{13} \mathrm{C}$ and ${ }^{15} \mathrm{O}(\beta+v){ }^{15} \mathrm{~N}$ decays in the most central regions where the $\mathrm{CN}$ cycle operates in nuclear equilibrium regime. The predictions for $\mathrm{CNO}$ neutrino fluxes are not very precise because the CNO fusion reactions have been not studied as efficiently as the pp reactions, since the Coulomb barrier is higher for the CNO reactions, implying a greater sensitivity to details of the solar model. The two neutrino fluxes $\Phi\left({ }^{13} \mathrm{~N}\right)$ and $\Phi\left({ }^{15} \mathrm{O}\right)$ depend almost linearly on the astrophysical S-factor $\mathrm{S}(\mathrm{E})$ of the ${ }^{14} \mathrm{~N}(\mathrm{p}, \gamma){ }^{15} \mathrm{O}$ reaction. Therefore, this reaction is important to precisely constrain the CNO cycle in the SSM.

An extensive study was pursued at the LUNA $400 \mathrm{kV}$ facility in the energy range 70-370 $\mathrm{keV}$ in the center of mass system with solid Ti-N targets and alternating a high resolution HP-Ge

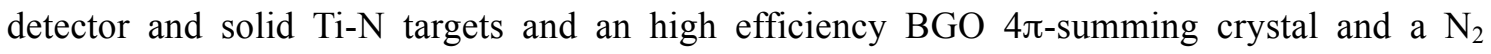
windowless gas target. The astrophysical factor obtained at LUNA [8,9] was a factor 0.6 lower than previously used and although this value has been anticipated based on older data but different extrapolation methods, the LUNA results settled the matter.

As a consequence, the CNO neutrino fluxes were decreased by the same factor and also the effect on the age of globular clusters, hence on the lower limit to the age of the Universe, was relevant: with the rate stellar evolutionaty models predicted a typical increase of 0.7-1.0 Gyr (with an uncertainity of $\pm 0.5 \mathrm{Gyr}$ ) for the ages of the universe.

The $400 \mathrm{kV}$ current LUNA accelerator and the unique low-background conditions of the underground LNGS laboratory are indeed perfectly suited to study most of the proton-capture reactions involved in the stellar $\mathrm{H}$ burning. On the other hand, a beam of higher energy is 
required to extend these studies to reactions between heavier isotopes, as those operating during more advanced phases of stellar evolution, namely the He and the $\mathrm{C}$ burnings.
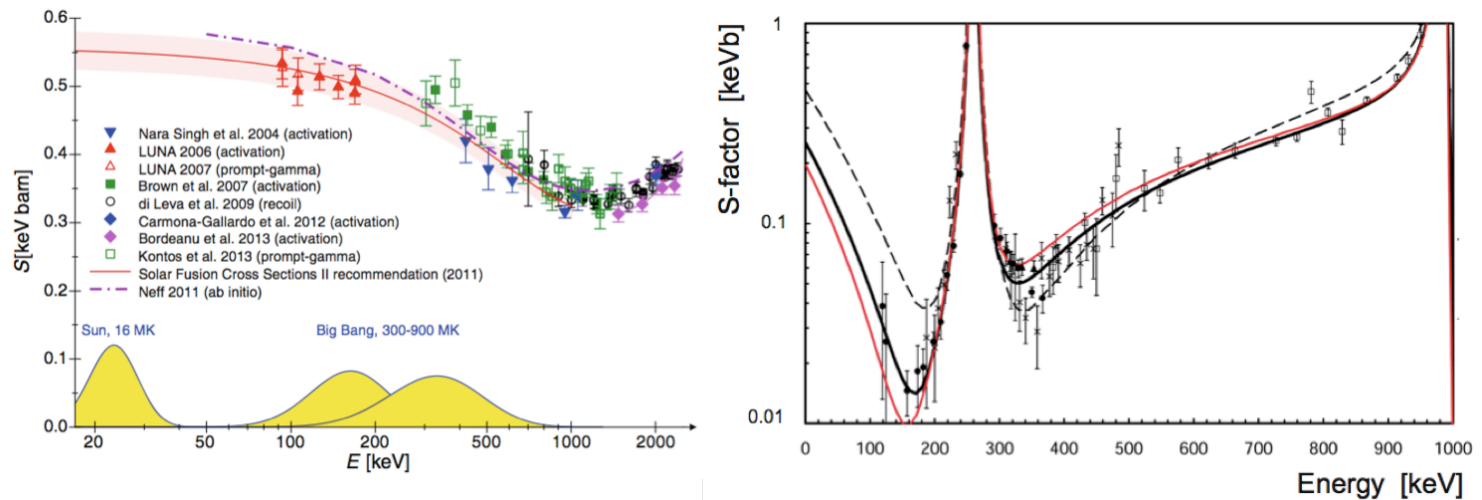

Fig.2 (Left) Overview of all existing measurements of the ${ }^{3} \mathrm{He}(\alpha, \gamma)^{7} \mathrm{Be}$ reaction S-factor; (Right) Astrophysical S-factor of ${ }^{14} N(p, \gamma)^{15} O$ for the ground state transition: the result of three different R-matrix fit are shown [3].

\section{The LUNA-MV project}

The LUNA MV project has been developed to overcome such a limit: a new 3.5 MV single-ended accelerator is going be installed in Hall B of Gran Sasso at the end of 2018 and it will provide hydrogen, helium and carbon (also doubly ionized) high current beams [1].

The new facility will be devoted to aggress the study of those key reactions of helium and carbon burning that determine and shape both the evolution of massive stars towards their final fate and the nucleosynthesis of most of the elements in the Universe.

As example, the ${ }^{12} \mathrm{C}(\alpha, \gamma){ }^{16} \mathrm{O}$ reactions that represent the "Holy Grail" of nuclear astrophysics since it determines the $\mathrm{C} / \mathrm{O}$ ratio left at the end of the $\mathrm{He}$ burning. This is a fundamental quantity affecting, for instance, white dwarf cooling timescale and the outcomes of both type Ia and core-collapse supernovae.

Another fundamental process is the ${ }^{12} \mathrm{C}+{ }^{12} \mathrm{C}$ fusion: the temperature at which $\mathrm{C}$ burning takes place depends on its rate, the larger the rate, the lower the $\mathrm{C}$-burning temperature and the stellar mass limit for $\mathrm{C}$ ignition. This limit separates the progenitors of white dwarfs, nova and type Ia supernovae, from those of core-collapse supernovae, neutron stars, and stellar mass black holes and therefore it controls the estimations of the expected numbers of these objects in a given stellar population, a quantity very relevant also to predict the possible collaps and gravitational wave emissions.

But even if more extensively studied, several important processes of H-burning are presently known at energies well above the Gamow peak: theoretical extrapolations to low energies are therefore unavoidable. The most popular methods are based on the R-matrix analyses [8] that for reliable extrapolations require nuclear physics informations obtained over a wide energy range.

As shown if Fig.2 (right) the cross-section of ${ }^{14} \mathrm{~N}(\mathrm{p}, \gamma){ }^{15} \mathrm{O}$ here displayed as the astrophysical S-factor for the ground-state transition is still insufficiently constrained by a good global fit of all available data [10]: this reaction has an uncertainty of about $8 \%$ when 
extrapolated to the solar Gamow peak. A new and comprehensive study of the ${ }^{14} \mathrm{~N}(\mathrm{p}, \gamma){ }^{15} \mathrm{O}$ reaction will be needed to cover a wide energy range.

A renewed effort will be pursued at LUNA and it will involve both LUNA accelerators: the existing LUNA $400 \mathrm{kV}$ machine as well as the future LUNA 3.5 MV facility. The combination of the two systems will allow to cover the necessary energy range with a sufficient overlap and without any hole between $200 \mathrm{keV}$ and $1.5 \mathrm{MeV}$. The ${ }^{14} \mathrm{~N}(\mathrm{p}, \gamma){ }^{15} \mathrm{O}$ reaction will allow also to perform the commissioning and the tuning of LUNA MV accelerator.

We remind that the ${ }^{14} \mathrm{~N}(\mathrm{p}, \gamma){ }^{15} \mathrm{O}$ is also very important in connection with the solar metallicity problem: it controls the speed of the whole $\mathrm{CNO}$ cycle since it proceeds with the slowest rate. Since the $\mathrm{CN}$-cycle keeps an almost linear dependence on the $\mathrm{C}+\mathrm{N}$ abundance in the core, the neutrino fluxes can be used as accurate tools to probe the total $\mathrm{CN}$ abundance. At present the error budget in the $\mathrm{C}+\mathrm{N}$ estimation is totally dominated by the uncertainties $(\sim 11 \%)$ in the cross section of the reactions ${ }^{7} \mathrm{Be}(\mathrm{p}, \gamma)^{8} \mathrm{~B}$ and ${ }^{14} \mathrm{~N}(\mathrm{p}, \gamma){ }^{15} \mathrm{O}$. Clearly, reducing the uncertainty of the latter reaction with LUNA MV will importantly contribute to set more stringent constraints on the central chemical composition of the Sun.

\section{Conclusions}

The cosmic silence of the underground LNGS laboratory and the $50 \mathrm{kV}$ and later on, the $400 \mathrm{kV}$ LUNA accelerators, have demostrated to be the perfect blend to push toward stellar energies the study of the proton-capture reactions involved in the stellar $\mathrm{H}$ burning. At the end of 2018, a new 3.5 MV single-ended accelerator will be installed in Hall B of Gran Sasso able to provide hydrogen, helium and carbon high current beams: this facility will open a new window to the study of helium and carbon burning key reactions that shape the evolution of massive stars towards their final fate and the nucleosynthesis of most of the elements in the Universe.

\section{References}

[1] https://luna.lngs.infn.it/

[2] A. Formicola et al., The LUNA II 400 kV accelerator, Nucl. Instr. and Meth. A507 (2003) 609

[3] A. Formicola, P. Corvisiero and G. Gervino, The nuclear physics of the hydrogen burning in the Sun, Eur. Phys. J. A52 (2016) 73

[4] V.N. Fetisov, Y.S. Kopysov, Nucl. Phys. A239 (1975) 511, W.A. Fowler, Nature. 238 (1972) 24

[5] R. Bonetti et al, Phys. Rev. Letter 82 (1999) 5205

[6] F. Confortola et al, Physical Review C 75 (2007) 065803

[7] A. Serenelli, Alive and well: A short review about stardard solar models, Eur. Phys. J. $\mathbf{A 5 2}$ (2016)73

[8] A. Formicola et al, Astrophysical S-factor of ${ }^{14} N(p, \gamma)^{15} O$, Phys. Lett. $\mathbf{B 5 9 1}$ (2004) 61

[9] M. Marta et al., The ${ }^{14} N(p, \gamma)^{15}$ O reaction studied with a composite detector, Phys. Rev. $\mathbf{C 8 3}$ (2011) 045804

[10] Q. Li et al., Cross Section of ${ }^{14} N(p, \gamma)^{15} O$ in the CNO cycle Phys. Rev. C93 (2016) 055806 